\title{
Population Pharmacokinetic and Pharmacodynamic Analysis of Belimumab Administered Subcutaneously in Healthy Volunteers and Patients with Systemic Lupus Erythematosus
}

\author{
Herbert Struemper ${ }^{1} \cdot$ Mita Thapar $^{2} \cdot$ David $_{\text {Roth }}^{3}$
}

Published online: 8 September 2017

(c) The Author(s) 2017. This article is an open access publication

\begin{abstract}
Background Intravenous belimumab $10 \mathrm{mg} / \mathrm{kg}$ every 4 weeks is indicated in patients with active, autoantibodypositive systemic lupus erythematosus receiving standard systemic lupus erythematosus care. Subcutaneous 200-mg weekly administration, which may prove more convenient for patients and improve adherence, is currently under investigation.

Objective The objective of this study was to characterize the population pharmacokinetics and exposure-efficacy response of subcutaneous belimumab in a pooled analysis of pharmacokinetic data [phase I: BEL114448 (NCT01583530) and BEL116119 (NCT01516450) in healthy subjects $(n=134)$; phase III: BEL112341 (NCT01484496) in adults with systemic lupus erythematosus $(n=554)]$ and pharmacodynamic data [BEL112341 in adults with systemic lupus erythematosus $(n=833)]$.
\end{abstract}

Electronic supplementary material The online version of this article (doi:10.1007/s40262-017-0586-5) contains supplementary material, which is available to authorized users.

Herbert Struemper

herbert.x.struemper@gsk.com

1 Quantitative Clinical Development, PAREXEL International on behalf of GlaxoSmithKline, 2520 Meridian Parkway, Suite 200, Durham, NC 27713, USA

2 ICON, Marlow, Buckinghamshire, UK

3 GlaxoSmithKline, Collegeville, PA, USA
Methods Non-linear mixed-effects modeling (NONMEM $\left.{ }^{\circledR}\right)$ was used to develop a population pharmacokinetic model and perform a covariate analysis. Subsequently, exploratory exposure-response analysis and logistic regression modeling was performed based on the individual parameter estimates of the population pharmacokinetic model.

Results Population-pharmacokinetic parameters for subcutaneous belimumab were consistent with those for intravenous belimumab and other immunoglobulin G1 monoclonal antibodies. Pharmacokinetic parameters and subcutaneous belimumab exposure were consistent between healthy subjects and patients with systemic lupus erythematosus, and no evidence for target-mediated disposition of belimumab was found. Subcutaneous belimumab steady-state exposure was achieved after $\sim 11$ weeks; subcutaneous belimumab steady-state minimum concentration exceeded that of intravenous belimumab after $<4$ weeks, and average steady-state concentration was similar to that achieved following intravenous administration. In patients with moderate-to-severe systemic lupus erythematosus, subcutaneous belimumab $200 \mathrm{mg}$ once weekly plus standard of care significantly improved the systemic lupus erythematosus responder index. However, at this dose, the systemic lupus erythematosus responder index response was not significantly associated with belimumab exposure concentrations.

Conclusion The analysis demonstrates that a 200-mg onceweekly dose of belimumab is appropriate for subcutaneous administration in patients with systemic lupus erythematosus and that no dose adjustments are required for adult patients to maintain efficacy and safety. 


\section{Key Points}

Population-pharmacokinetic parameters for subcutaneous belimumab were consistent with those immunoglobulin G1 monoclonal antibodies.

In patients with moderate-to-severe systemic lupus erythematosus, subcutaneous belimumab (200 mg, weekly) plus standard systemic lupus erythematosus care significantly improved the systemic lupus erythematosus responder index. However, at this dose, the systemic lupus erythematosus responder index response was not significantly associated with belimumab exposure concentrations.

No dose adjustments are required for subcutaneous belimumab in adult patients with systemic lupus erythematosus to maintain efficacy and safety. for intravenous belimumab and other

patient convenience and independence, and reduce the overall healthcare burden of belimumab treatment [10-14]. The ability to administer treatments away from the clinic can largely eliminate substantial time and financial costs associated with travel to/from the drug-administering site, for the infusion itself, and for post-infusion monitoring. In a study of patients with rheumatoid arthritis, more patients chose SC treatment than IV treatment, with the reduced need to travel to receive an infusion being an influential factor [15]. A popPK model comparing IV with SC belimumab has previously been developed using pooled data from two phase I studies (BEL114448 [NCT01583530] and BEL116119 [NCT01516450]) in healthy subjects [12-14]. This present analysis characterizes the popPK and exposure-efficacy response of belimumab following SC administration in a pooled analysis of PK data from these aforementioned phase I studies in healthy subjects $[12,14]$, combined with PK and pharmacodynamics (PD) data from a large phase III study in adult patients with SLE [BEL112341 (NCT01484496)] [10].

\section{Methods}

\section{Introduction}

Systemic lupus erythematosus (SLE) is a chronic multisystem autoimmune disease presenting a broad spectrum of clinical manifestations [1]. B-lymphocyte stimulator (BLyS) is a member of the tumor necrosis factor family, and plays an integral role in the selection and survival of $B$ cells [2]. Although the definitive role of BLyS in SLE has not yet been fully elucidated, B cells and BLyS have been identified as playing critical roles in SLE pathogenesis [2].

Belimumab is a recombinant, human, immunoglobulin G1 (IgG1) monoclonal antibody that targets BLyS, inhibiting B-cell survival and differentiation without directly causing B-cell death [2, 3]. Two phase III, randomized, double-blind, placebo-controlled studies [BLISS52 (NCT00424476) and BLISS-76 (NCT00410384)] have demonstrated that intravenous (IV) belimumab plus standard SLE care is associated with a significant reduction in disease activity in active autoantibody-positive SLE [4, 5].

The IV formulation of belimumab is indicated for the treatment of active autoantibody-positive SLE in patients receiving standard SLE care, at a dose of $10 \mathrm{mg} / \mathrm{kg}$ at 2-week intervals for the first three doses and every 4 weeks thereafter [6]. A population-pharmacokinetic (popPK) model for IV belimumab was developed [7] using pooled data from phase I and II studies [8, 9] and the two pivotal phase III studies, BLISS-52 and BLISS-76 [4, 5].

A subcutaneous (SC) formulation of belimumab is currently under investigation, the use of which may improve

\subsection{Objectives}

The objectives of the popPK analysis were to develop a model that characterizes the $\mathrm{PK}$ disposition of $\mathrm{SC}$ belimumab following 200-mg weekly (QW) administration in healthy subjects and patients with SLE, to evaluate the potential effect of selected covariates on key PK parameters, and to estimate individual subject PK parameters such as steady-state area under the curve, average steady-state concentration $\left(C_{\mathrm{avg}}\right)$, steady-state maximum concentration, steady-state minimum concentration $\left(C_{\min }\right)$, elimination half-life for the first phase, elimination half-life for the terminal phase, and steady-state volume of distribution. In addition, an exploratory exposure-response analysis was proposed, plotting exposure $\left(C_{\text {avg }}\right)$ vs. the selected PD/efficacy/safety endpoints at week 52, alongside a popPK/PD analysis on a selected PD/efficacy/safety endpoint(s) of special interest (phase III study only).

\subsection{Study Design}

In this popPK/PD analysis, PK data from 688 individuals from three studies [phase I: BEL116119 and BEL114448 ( $n=134)$; phase III: BEL112341 [10] (NCT01484496; $n=554)$ ] and PD data from BEL112341, for which the methods and results have been described previously [10, 12, 14], were analyzed using a popPK/PD approach (Table 1). The primary efficacy endpoint for Study BEL112341 was the SLE responder index 4 (SRI4), a 
Table 1 Studies included in the belimumab population pharmacokinetic/pharmacodynamic (PK/PD) analysis

\begin{tabular}{|c|c|c|c|c|}
\hline Study & Population & Dose and administration & Planned PK data & $\begin{array}{l}\text { PD/efficacy/safety } \\
\text { endpoints }\end{array}$ \\
\hline $\begin{array}{l}\text { Phase I } \\
\text { (BEL116119 } \\
\text { [12]) }\end{array}$ & $\begin{array}{l}n=16 \\
\text { Healthy } \\
\text { volunteers } \\
\text { (Japan) }\end{array}$ & $\begin{array}{l}\text { Two single-dose groups } \\
\text { IV group: } 200 \text {-mg single- } \\
\text { dose IV infusion over } 1 \mathrm{~h} \\
(n=8) \\
\text { SC group: } 200-\mathrm{mg} \mathrm{SC} \\
\text { single dose } \\
\text { over } 10-15 \mathrm{~s}(n=8)\end{array}$ & $\begin{array}{l}\text { SC group: pre-dose (day } 0), 6 \mathrm{~h} \text {, days } 1,2,3,4,5,6 \text {, } \\
\quad 7,10,14,21,28,42,56, \text { and } 70 \text { post-dose }\end{array}$ & Not available \\
\hline $\begin{array}{l}\text { Phase I } \\
\text { (BEL114448) }\end{array}$ & $\begin{array}{l}n=118 \\
\text { Healthy } \\
\text { volunteers } \\
\text { (USA) }\end{array}$ & $\begin{array}{l}\text { Four single-dose groups } \\
\text { Group 1: SC, } 2 \text { sequential } \\
\text { injections }(0.6 \mathrm{~mL} / \\
\text { injection; } 200 \mathrm{mg} / \mathrm{mL}) \\
\text { Group 2: SC, } 1 \text { injection } \\
(1.2 \mathrm{~mL} / \text { injection; } \\
200 \mathrm{mg} / \mathrm{mL}) \\
\text { Group 3: SC, } 1 \text { injection } \\
\text { (1.2 mL/injection; } \\
200 \mathrm{mg} / \mathrm{mL}) \\
\text { Group 4: IV control } \\
\text { (240 mg lyophilized } \\
\text { formulation) } \\
\text { Two groups with 4-weekly } \\
\text { SC doses } \\
\text { Group 5: weekly } \times 4 ; \mathrm{SC} \text {, } \\
2 \text { sequential injections } \\
(0.6 \mathrm{~mL} / \text { injection; } \\
200 \mathrm{mg} / \mathrm{mL}) \\
\text { Group 6: weekly } \times 4 ; \mathrm{SC} \text {, } \\
1 \text { injection }(1.2 \mathrm{~mL} / \\
\text { injection; } 200 \mathrm{mg} / \mathrm{mL})\end{array}$ & $\begin{array}{l}\text { Groups } 5 \text { and } 6 \text { : pre-dose (day } 0 \text { ), } 6 \mathrm{~h} \text {, days } 1,2,3 \text {, } \\
4,5,6,7 \text { (prior to second dosing), } 14 \text { (prior to } \\
\text { third dosing), } 21 \text { (prior to fourth dosing), } 6 \text { h post- } \\
\text { fourth dose, days } 22,23,24,25,26,27,28,31,35 \text {, } \\
42,49,63,77,91 \text {, and } 119 \text { post-dose }\end{array}$ & Not available \\
\hline $\begin{array}{l}\text { Phase III } \\
\text { (BEL112341) }\end{array}$ & $\begin{array}{l}n=833(554 \text { on } \\
\text { belimumab; } \\
279 \text { on } \\
\text { placebo) } \\
\text { Patients with } \\
\text { SLE (global) }\end{array}$ & $\begin{array}{l}\text { SC } 200 \mathrm{mg} \text {, weekly for } \\
51 \text { weeks }\end{array}$ & $\begin{array}{l}\text { Pre-dose (day } 0 \text { ), weeks } 4,8,16,24 \text {, and } 52 \text {, } \\
1-4 \text { weeks after the last dose (for patients exiting } \\
\text { before week } 52 \text { ), } 8 \text { weeks after the last dose (for } \\
\text { patients not entering the extension phase or } \\
\text { withdrawing from the study at any time) }\end{array}$ & $\begin{array}{l}\text { SRI response } \\
\text { Change in SELENA- } \\
\text { SLEDAI score } \\
\text { Change in naïve } \\
\text { peripheral B-cell } \\
\text { subset (CD20+l } \\
\text { CD27-) } \\
\text { Change in } \\
\text { complement } \\
\text { (C3, C4) level } \\
\text { Change in anti- } \\
\text { dsDNA antibody } \\
\text { level } \\
\text { Change in total serum } \\
\text { IgG level } \\
\text { Frequency of SAEs } \\
\text { Frequency of } \\
\text { serious/severe } \\
\text { infections }\end{array}$ \\
\hline
\end{tabular}

$C D$ cluster of differentiation, $d s D N A$ double-stranded DNA, $I g G$ immunoglobulin G, $I V$ intravenous, $S A E s$ serious adverse events, $S C$ subcutaneous, SELENA-SLEDAI Safety of Estrogens in Lupus Erythematosus National Assessment-Systemic Lupus Erythematosus, SLE systemic lupus erythematosus, $S R I$ systemic lupus erythematosus responder index 
composite index that includes a $\geq 4$-point reduction in the Safety of Estrogens in Lupus Erythematosus National Assessment-SLE Disease Activity Index (SELENA-SLEDAI) [10].

All subjects with evaluable dosing, actual sampling time, and belimumab concentration data were included in the popPK dataset and analysis. All subjects and placebo subjects with evaluable PD efficacy/safety data were included in the popPK/PD dataset and analysis.

\subsubsection{Assay Methods}

Serum concentrations of belimumab were determined by a validated electrochemiluminescence-based immunoassay with a lower limit of quantitation of $100 \mathrm{ng} / \mathrm{mL}$ of belimumab in human serum.

\subsection{Modeling}

\subsubsection{Software and Estimation Methods}

Models were developed with a non-linear mixed-effects modeling approach using NONMEM ${ }^{\circledR}$ Version VII level 3.0 (ICON Development Solutions, Ellicott City, MD, USA) and PDx-Pop (Version 5.1). Population pharmacokinetic parameters were estimated using the first-order conditional estimation method with interaction; popPK/PD (logistic regression) parameters were estimated using the Laplace estimation method.

\subsubsection{Population Pharmacokinetic Model Development}

A linear two-compartment model (ADVAN3, TRANS4 subroutine) was chosen as the structural PK model, based on the previously characterized IV popPK model [7], and two-compartment behavior was confirmed via exploratory data analysis of the serially sampled IV/SC PK data. Firstorder absorption into the central compartment with rate constant and a lag time was added to model the SC data. Inter-individual variability was tested and included with lognormal random-effect distributions. A hybrid full model/backward elimination approach was used to develop the covariate model. The full model was determined by a selection of 26 covariate effects guided by the covariate analysis for the IV popPK analysis. The full model was reduced to the final model by step-wise removal (backward elimination) of effects not meeting the statistical significance criterion ( $\alpha=0.001$, as for IV popPK analysis).

The model was qualified with diagnostic plots, visual predictive checks (500 data sets), and with a non- parametric bootstrap analysis (500 datasets, random sampling with replacement with subjects as the sampling unit). Following popPK model development, PK profiles were simulated using population parameters from this analysis for the SC curves (Table 3) and from the IV popPK model [7] for the IV curves. Steady-state parameters were derived from profiles following 24 weeks of dosing.

\subsubsection{Population Pharmacokinetic/Pharmacodynamic Model Development}

Following a graphical exposure-response analysis for selected PD/efficacy/safety endpoints, a logistic regression model was developed to characterize SRI4 response in relation to $C_{\text {avg }}$ (generally considered relevant for both efficacy and safety dose response) and other subject characteristics as potential predictors of efficacy. The probability of being a SRI responder was obtained by the equation:

$P=\exp (L P) /(1+\exp (L P))$.

When the observed response is 0 , the variable to be fitted is $Y=1-P$ and when the observation is $1, Y=P$.

The main logistic regression analysis was applied to the combined placebo and belimumab-treated SRI data set.

Consistent with covariate model development of the popPK model, this model was developed using a hybrid full model/backward elimination approach to identify significant predictors $(\alpha=0.05)$. The full covariate model included the effects of 18 predictors including demographic variables, baseline disease characteristics, presence of co-medications, and belimumab exposure (difference of $C_{\text {avg }}$ to median $C_{\text {avg }}$ in the belimumab-treated population). To characterize the SRI response in belimumab-treated patients, these covariate effects were only applied to belimumab-treated patients; the response for placebo patients being represented by the intercept parameter. The final model was derived by stepwise backwards elimination of effects not meeting the significance criterion for the loglikelihood ratio test.

In a sensitivity analysis to explore any impact of placebo data on the effects retained in the final model of the main analysis, an alternate analysis was conducted by restricting the exposure-response data to belimumabtreated patients only, i.e., removing data for placebo patients. The final model of the sensitivity analysis was derived by constructing a full model with the same covariate effects (except for the treatment effect) as in the main analysis and applying the same stepwise backwards elimination procedure to arrive at an alternate final model. 
Table 2 Subject demographics and baseline characteristics (population-pharmacokinetic analyses: $N=688$ )

\begin{tabular}{|c|c|c|}
\hline \multicolumn{3}{|l|}{ Demographics } \\
\hline Sex: female, $n(\%)$ & & $584(85)$ \\
\hline \multicolumn{3}{|l|}{ Race, $n(\%)$} \\
\hline White & & $422(61)$ \\
\hline Asian & & $137(23.5)$ \\
\hline African American & & $76(11)$ \\
\hline American Indian or Alaska $\mathrm{N}$ & Jative & $41(6)$ \\
\hline Other & & $12(1.7)$ \\
\hline Mean (SD) age, years & & $37.6(11.8)$ \\
\hline Median (range) & & $37(18-77)$ \\
\hline Mean (SD) weight, kg & & $70.0(17.6)$ \\
\hline Median (range) & & $67.0(34.1-138)$ \\
\hline Mean (SD) BMI, $\mathrm{kg} / \mathrm{m}^{2}$ & & $26.1(6.18)$ \\
\hline Median (range) & & $24.7(14.8-72.7)$ \\
\hline Characteristics & Mean (SD) & Median (range) \\
\hline $\mathrm{IgG}, \mathrm{g} / \mathrm{L}$ & $14.9(5.43)$ & $13.7(4.70-53.5)$ \\
\hline Proteinuria, mg/mg ${ }^{\mathrm{a}}$ & $0.340(0.651)$ & $0.118(0.0210-6.23)$ \\
\hline Albumin levels, $g / L$ & $40.7(4.47)$ & $41.0(18.0-55.0)$ \\
\hline Creatinine clearance, $\mathrm{mL} / \mathrm{min}$ & $115(38.3)$ & $109(35.0-353)$ \\
\hline Hemoglobin, g/L & $126(15.8)$ & $126(73.0-173)$ \\
\hline WBC count, Gi/L & $6.09(2.41)$ & $5.70(1.30-13.9)$ \\
\hline
\end{tabular}

$B M I$ body mass index, $\operatorname{Ig} G$ immunoglobulin $\mathrm{G}, S D$ standard deviation, $W B C$ white blood cell

${ }^{a}$ Study BEL112341 determined proteinuria by measuring the spot urine protein:creatinine ratio (mg/mg). In the BEL112341 clinical study report and other submission documents, these values are reported as $\mathrm{g} / 24-\mathrm{h}$ equivalent proteinuria values. In Study BEL112341, 99 subjects (18\% of subjects in Study BEL112341) had proteinuria $>0.5 \mathrm{~g} / 24 \mathrm{~h}$ and 19 subjects $(3 \%$ of subjects in Study BEL112341) had proteinuria $\geq 2 \mathrm{~g} / 24 \mathrm{~h}$

\section{Results}

\subsection{Population-Pharmacokinetic Model}

\subsubsection{Subject Disposition}

A total of 4958 belimumab concentrations (on average, seven per subject) from 688 healthy volunteers and patients with SLE were included in the popPK analysis (Table 2). Of these 4958 concentrations, 2298 were contributed by 134 subjects who were serially sampled [17 concentrations per subject on average, from healthy volunteers in two phase I studies (BEL116119 [12] and BEL114448 [14])] and 2660 concentrations were contributed by 554 patients who were sparsely sampled [five concentrations per patient on average, from patients with SLE in one phase III study (BLISS SC BEL112341 [10])]. Quantifiable pre-first dose concentrations from six subjects were excluded from the analysis. Study-specific median values were imputed for missing continuous covariate values in the dataset.

\subsubsection{Population Pharmacokinetic Modeling}

The pharmacokinetics of SC belimumab was best described by a linear two-compartment base model with an absorption lag time followed by first-order absorption into the central compartment and elimination from the central compartment. No evidence for substantial target-mediated disposition of belimumab was found. The random-effects structure of the model included inter-individual variability on central clearance (CL), inter-compartmental clearance $(Q)$, central $\left(V_{\mathrm{c}}\right)$ and peripheral $\left(V_{\mathrm{p}}\right)$ volumes of distribution, as well as a combined proportional and additional residual error model.

Significant covariate effects $(\alpha=0.001)$ retained in the final model after backward elimination included body mass index (BMI) on $V_{\mathrm{c}}$ (power coefficient of -0.6110) and body weight on $\mathrm{CL}$ and $V_{\mathrm{c}}$ (power coefficients of 0.75 and 1.00, respectively), and albumin (Fig. 1a) and $\mathrm{IgG}$ (Fig. 1b) on CL. In general, belimumab CL increased with increasing levels of baseline $\operatorname{IgG}$ (power coefficient of 0.347). An inverse relationship was seen between baseline albumin levels and belimumab CL, whereby CL decreased with albumin (power coefficient of -0.736). The only pharmacokinetically relevant covariates (defined as a change relative to the population median beyond the -20 to $+25 \%$ range in CL or $V_{\mathrm{c}}$ for the 5th to 95th percentile range of the patient covariate distribution) were BMI and body weight. The predicted effects of age, sex, race, disease activity, concomitantly administered medications (corticosteroids, angiotensin-converting enzyme inhibitors, azathioprine, methotrexate, mycophenolate mofetil, antimalarial medications, statins, non-steroidal anti-inflammatory drugs, and aspirin), baseline characteristics, or other tested factors were neither statistically significant nor pharmacokinetically relevant (the exposure-response analysis demonstrated that the SRI response was at the 200-mg QW dose level, which is not significantly correlated with exposure variability). Pharmacokinetic parameters and SC belimumab exposure were consistent between healthy subjects and patients with SLE.

The parameters of the final SC belimumab popPK model were estimated with good precision (relative standard error $\%$ 0.3-28\%) and agreement between model and bootstrap parameters (Table 3). Goodness-of-fit plots for the final model indicate that the model provided an appropriate description of the data (Fig. 2). Visual predictive checks indicated that the final model represented both central tendency and variability of belimumab pharmacokinetics well for both routes of administration (Fig. 3) and for each 


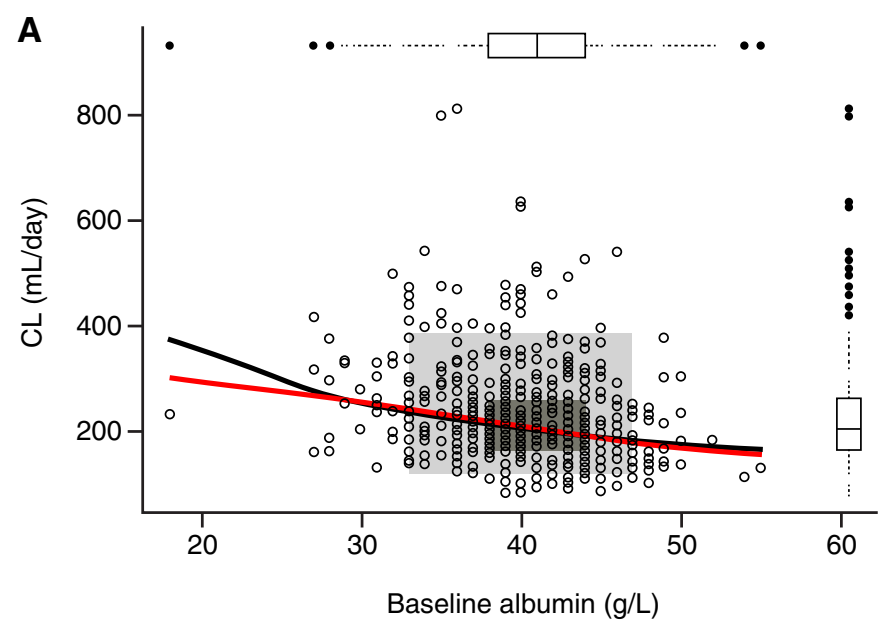

Fig. 1 Covariate effect of baseline albumin (a) and immunoglobulin $\mathrm{G}$ [IgG] (b) on clearance. Margin box plots: black bar median; box interquartile range; circles outliers. The red line indicates the locally weighted scatterplot smoothing fit of observed data; the black line

of the three studies [Fig. 2 of the Electronic Supplementary Material (ESM)], the latter indicating that the model appropriately represents both healthy subjects and patients with SLE.

Based on the popPK parameters, belimumab is absorbed following SC administration with a lag time of 0.18 days, absorption half-life of 3 days, and bioavailability of $74 \%$. Key structural popPK parameters included total systemic $\mathrm{CL}$ of $204 \mathrm{~mL} /$ day; $V_{\mathrm{c}}$ and volume of distribution of the peripheral compartment ( 2300 and $2650 \mathrm{~mL}$, respectively); and inter-compartmental CL of $698 \mathrm{~mL} /$ day (Table 3). A terminal half-life of 18 days, distribution half-life of 1.1 days, and steady-state volume of distribution of $4950 \mathrm{~mL}$ were derived from the structural parameters.

To compare chronic exposures resulting from the IV dosing regimen approved for SLE with the SC phase III dosing regimen, $\mathrm{PK}$ profiles based on the popPK parameters of the IV [7] and SC models were simulated (Fig. 4, solid line). First-dose and steady-state time to maximum concentration for SC belimumab were shown to be 5.5 and 2.6 days, respectively. Steady-state exposure for SC belimumab was reached after approximately 11 weeks of 200-mg QW dosing (at $\geq 11$ weeks $C_{\min }$ exceeds $95 \%$ of steady state $C_{\mathrm{min}}$ ). Steady-state area under the curve from pre-dose to the end of the dosing interval for SC belimumab 200-mg QW dosing was 726 day $\times \mu \mathrm{g} / \mathrm{mL}$, and steady-state maximum concentration, $C_{\mathrm{min}}$, and $C_{\text {avg }}$ were 108, 97, and $104 \mu \mathrm{g} / \mathrm{mL}$, respectively (Fig. 4). Subcutaneous belimumab $C_{\min }$ exceeded that of IV belimumab steady-state $C_{\min }$ after $<4$ weeks, and $C_{\mathrm{avg}}$ of $\mathrm{SC}$ belimumab was similar to that achieved following IV administration (Fig. 4).

To illustrate how the impact of body weight and BMI on chronic exposure differs between weight-proportional IV

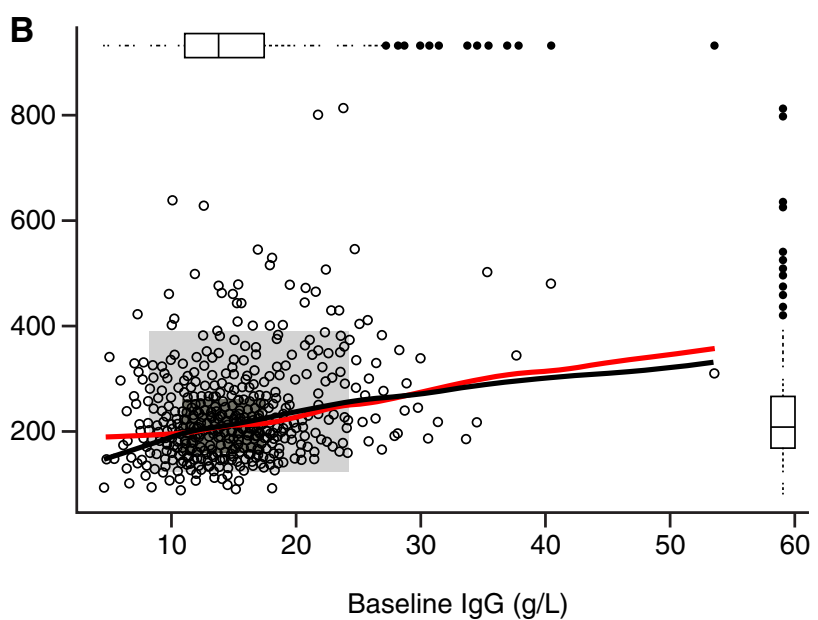

indicates the predicted relationship; the light gray rectangle indicates the 5th to 95th percentiles; and the dark gray rectangle indicates the interquartile range

and fixed SC dosing, two additional PK profiles for a lowweight and heavy subject were simulated by setting both body weight and BMI to the 5th and 95th percentiles, respectively, in Study BEL112341 (Fig. 4, dotted and dashed lines, respectively, other covariates were kept at median values). As expected, increased body size resulted in a smaller exposure for SC belimumab and a larger exposure for IV belimumab (and vice versa), while the range of deviation from the prototypical popPK profile was comparable for both routes of administration. Figure 3 of the ESM illustrates this relationship by comparing individual $C_{\text {avg }}$ estimates for this and the IV popPK analysis [7]: geometric means and ranges of $C_{\text {avg }}$ are well matched between SC 200-mg QW dosing and IV $10-\mathrm{mg} / \mathrm{kg}$ every 4 weeks dosing with reversed trends in relation to body weight.

\subsection{Exposure-Response Analysis}

\subsubsection{Patient Disposition}

A total of 833 patients (554 on belimumab and 279 on placebo; all from Study BEL112341) had SRI response values that were included in the popPK/PD analysis. In the belimumab arm, 340/554 (61\%) patients were responders and 214/554 (39\%) were non-responders; in the placebo arm, 135/279 (48\%) patients were responders and 144/279 (52\%) were non-responders.

\subsubsection{Graphical Exposure-Response Analysis}

Selected efficacy (SRI, change from baseline in SELENASLEDAI at week 52), safety (presence of serious adverse events, presence of serious infections), and PD data 
Table 3 Parameter estimates of the final population-pharmacokinetic model

\begin{tabular}{|c|c|c|c|}
\hline Parameter & Implementation & Point estimate $\left(\% \mathrm{RSE}^{\mathrm{a}}, 95 \% \mathrm{CI}\right)$ & Bootstrap estimates, median (\%RSE, 95\% CI) \\
\hline \multicolumn{4}{|l|}{ Fixed effects } \\
\hline F [fraction] & THETA (6) & $0.742(6.75,0.644-0.840)$ & $0.742(5.1,0.673-0.824)$ \\
\hline ALAG [day] & THETA (7) & $0.179(1.99,0.172-0.186)$ & $0.179(3.2,0.168-0.189)$ \\
\hline Kabs [1/day] & THETA (5) & $0.235(5.74,0.209-0.261)$ & $0.232(9,0.198-0.282)$ \\
\hline CL [mL/day] & THETA (1) & $204(6.76,177-231)$ & $204(5.0,186-226)$ \\
\hline Effect of BWT [kg] & $\times(\mathrm{BWT} / 67)^{0.75}$ & - & - \\
\hline Effect of BALB [g/L] & $\times(\mathrm{BALB} / 41)^{\theta}$ & $-0.736(14.1,-0.940$ to -0.532$)$ & $-0.737(14.6,-0.953$ to -0.533$)$ \\
\hline Effect of BIGG [g/L] & $\times(\mathrm{BIGG} / 13.7)^{\theta}$ & $0.347(10.4,0.276-0.418)$ & $0.352(11.7,0.272-0.436)$ \\
\hline$V_{\mathrm{c}}[\mathrm{mL}]$ & THETA (2) & $2300(9.83,1860-2740)$ & $2290(8.9,1890-2705)$ \\
\hline Effect of BWT & × (BWT/67) & - & - \\
\hline Effect of BBMI $\left[\mathrm{kg} / \mathrm{m}^{2}\right]$ & $\times(\mathrm{BBMI} / 24.7)^{\theta}$ & $-0.610(28.2,-0.947$ to -0.273$)$ & $-0.608(36.7,-0.997$ to -0.16$)$ \\
\hline$Q[\mathrm{~mL} / \mathrm{day}]$ & THETA (3) & $698(13.3,517-879)$ & $683(22.1,494-1115)$ \\
\hline Effect of BWT & $\times(\mathrm{BWT} / 67)^{0.75}$ & - & - \\
\hline$V_{\mathrm{p}}[\mathrm{mL}] 0.8$ & THETA (4) & $2650(7.09,2280-3020)$ & $2650(6.8,2360-3045)$ \\
\hline Effect of BWT & × (BWT/67) & - & - \\
\hline \multicolumn{4}{|l|}{ Inter-individual variability } \\
\hline$\omega_{\mathrm{CL}}^{2}$ & OMEGA $(1,1)$ & $0.0910(6.11,0.0801-0.102)$ & $0.0901(8.1,0.077-0.105)$ \\
\hline$\omega_{\mathrm{V} 1}^{2}$ & OMEGA $(2,2)$ & $0.497(9.94,0.400-0.594)$ & $0.488(16.9,0.316-0.650)$ \\
\hline$\omega_{\mathrm{CL} / \mathrm{V} 1}^{2}$ & OMEGA $(2,1)$ & $0.0630(19.7,0.0387-0.0873)$ & $0.0632(749.5,0.013-0.103)$ \\
\hline$\omega_{\mathrm{Q}}^{2}$ & OMEGA $(3,3)$ & $1.07(21.1,0.627-1.51)$ & $1.03(46.4,0.206-1.892)$ \\
\hline$\omega^{2} \mathrm{v} 2$ & OMEGA $(4,4)$ & $0.110(16.5,0.0743-0.146)$ & $0.111(19.9,0.076-0.166)$ \\
\hline \multicolumn{4}{|l|}{ Residual variability } \\
\hline$\sigma_{\text {proportional }}^{2}$ & SIGMA (1) & $0.0327(2.63,0.0310-0.0344)$ & $0.0328(6.6,0.029-0.0375)$ \\
\hline$\sigma_{\text {additive }}^{2}$ & SIGMA (2) & $0.134(23.7,0.0717-0.196)$ & $0.138(39.1,0.056-0.291)$ \\
\hline
\end{tabular}

$A L A G$ absorption lag time, $B A L B$ baseline albumin level, $B B M I$ baseline body mass index, $B I G G$ baseline immunoglobulin $\mathrm{G}$ level, $B W T$ baseline body weight, $C I$ confidence interval, $C L$ systemic clearance, $F$ bioavailability for SC dosing, Kabs absorption rate constant, $Q$ intercompartmental clearance, RSE relative standard error, $S C$ subcutaneous, THETA or $\theta$ corresponding structural model parameter, $V c$ central volume of distribution, $V_{p}$ peripheral volume of distribution

${ }^{\text {a }}$ Calculated as (standard error/mean) $\times 100 \%$

(change from baseline of naïve peripheral B cells, complement $\mathrm{C} 3$, complement $\mathrm{C} 4$, anti-double-stranded DNA antibody level, and $\mathrm{IgG}$ at week 52) were graphically examined for a relationship with SC belimumab exposure $\left(C_{\text {avg }}\right)$. For the safety endpoints, the $C_{\text {avg }}$ interquartile ranges of subjects with serious adverse events or infections contained the interquartile ranges of subjects without such events (Fig. 1 of the ESM). Similarly, none of the other endpoints showed a strong consistent correlation with SC belimumab exposure (data not shown).

\subsubsection{Systemic Lupus Erythematosus Responder Index Logistic Regression Modeling}

To identify any predictors of efficacy and to further explore (the lack of) exposure effects on efficacy, a logistic regression analysis of the SRI, the primary efficacy endpoint, was conducted. The starting point was a logistic regression model with the following candidate predictors applied to belimumab-treated patients: sex, age, race categories, body weight quartiles, SELENA-SLEDAI score, anti-double-stranded DNA antibody presence, low complement, proteinuria, belimumab treatment, difference to median belimumab steady-state $C_{\text {avg }}$, corticosteroid treatment, antimalarial treatment, and immunosuppressant treatment.

After reducing the full model to statistically significant predictors $(\alpha=0.05)$, only the following effects remained in the final model (Table 4). A baseline SELENA-SLEDAI score $\geq 10$ or American Indian/Alaska Native ethnicity was found to increase the probability of being an SRI responder for patients treated with belimumab; baseline proteinuria $>0.5 \mathrm{~g} / 24 \mathrm{~h}$ or African American ethnicity decreased the probability of being an SRI responder for these patients (Fig. 5). For patients with SLE receiving belimumab $200 \mathrm{mg}$ QW, exposure variations were not a significant predictor of SRI response, whether the exposure effect was implemented linearly (change in objective function 
Fig. 2 Goodness-of-fit plots for the final population-

pharmacokinetic model. Blue circles indicate the observed/ predicted belimumab concentrations; black lines indicate the identity or zero lines; and red lines indicated the locally weighted scatterplot smoothing line
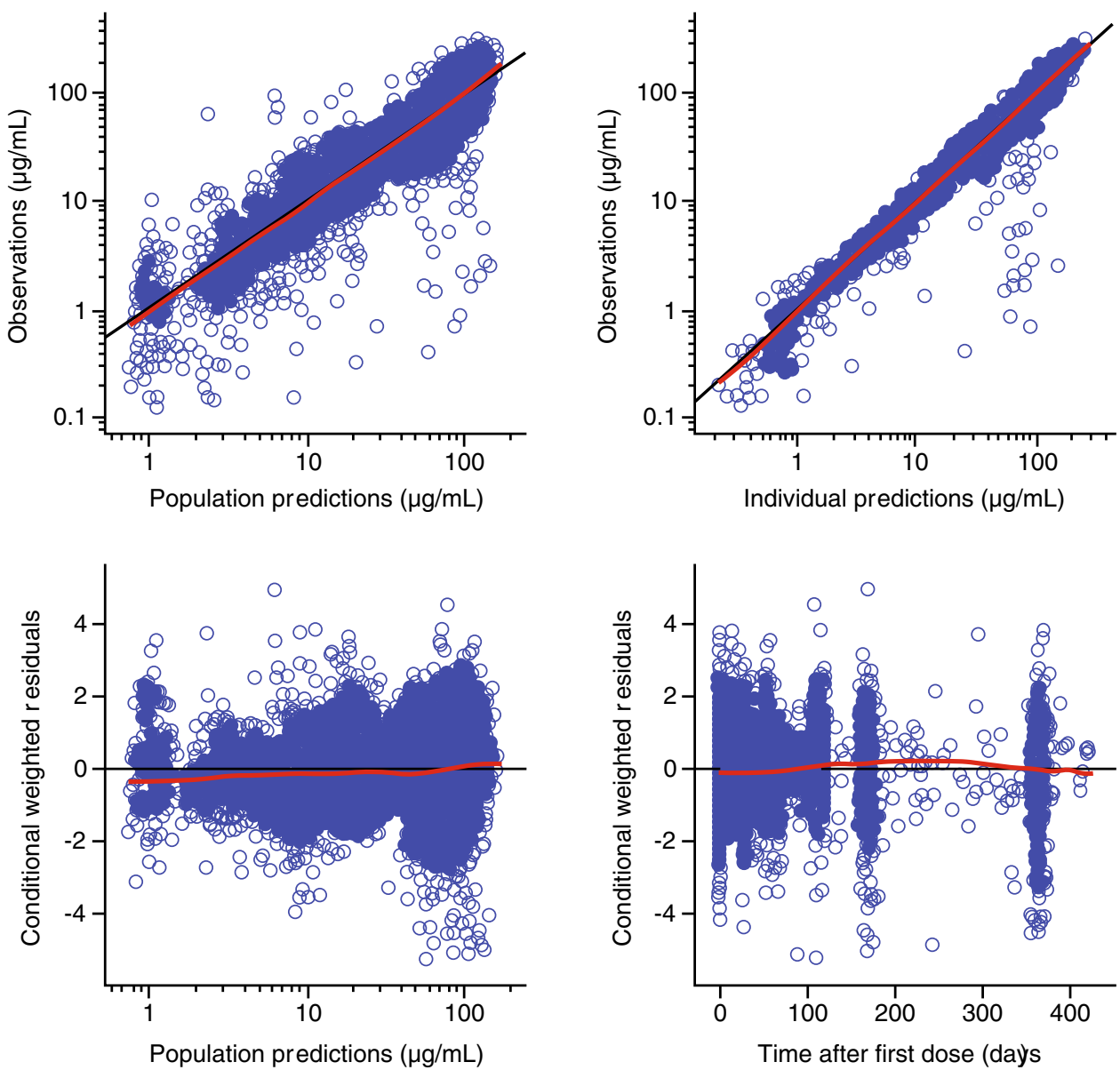

Fig. 3 Visual predictive check for final population-pharmacokinetic model stratified by route of administration. Open circle observed concentrations; solid line median of observed concentrations; dashed lines 5th and 95th percentile of observed concentration. The red shaded region indicates the $95 \%$

prediction interval for the median of predicted concentrations and the blue shaded regions indicated the 95\% prediction interval for the 5 th and 95 th percentiles of predicted concentrations. $I V$ intravenous, $S C$ subcutaneous

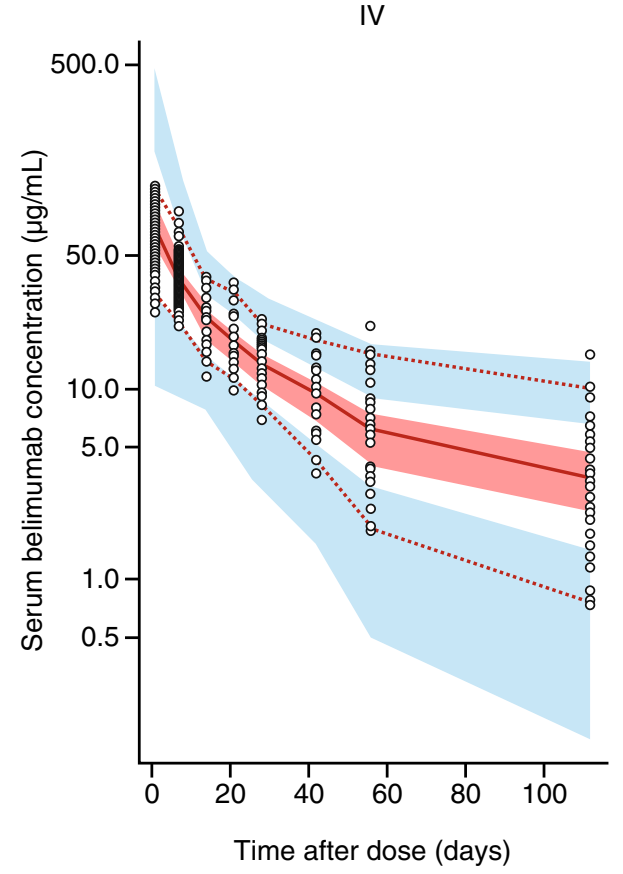




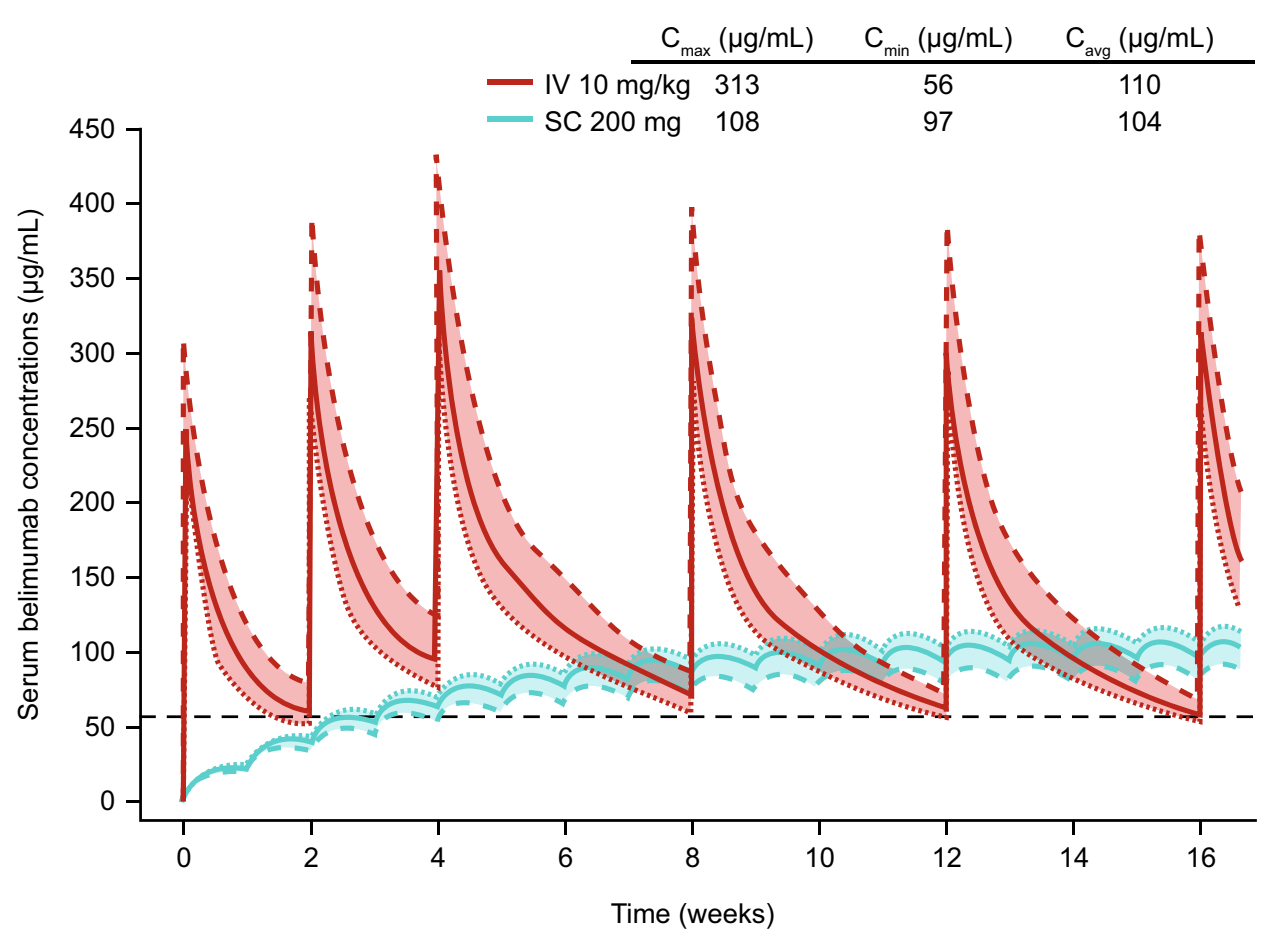

Fig. 4 Comparison of pharmacokinetic (PK) profiles and steady-state PK parameters for intravenous (IV) belimumab $10 \mathrm{mg} / \mathrm{kg} 3 \times$ every 2 weeks $(\mathrm{Q} 2 \mathrm{~W})$ then every 4 weeks $(\mathrm{Q} 4 \mathrm{~W})$ vs. subcutaneous (SC) belimumab $200 \mathrm{mg}$ once weekly (QW) based on simulations with population-PK (popPK) parameters. Red or blue solid lines represent deterministic simulations based on popPK parameters from IV [7] and SC (Table 3) popPK analyses; red or blue dotted/dashed lines represent PK profiles based on identical popPK parameters except for setting body weight and body mass index (BMI) parameters to the 5 th and 95th percentiles of body weight $(46.6$ and $105.1 \mathrm{~kg}$ ) and BMI (18.4 and $38.5 \mathrm{~kg} / \mathrm{m}^{2}$ ) in Study BEL112341; and the black dashed line represents steady-state minimum (trough) concentration $\left(C_{\mathrm{min}}\right)$ for chronic IV dosing. $C_{\text {avg }}$ average steady-state concentration, $C_{\max }$ maximum concentration
Table 4 Parameter estimates of the final logistic regression model

\begin{tabular}{llll}
\hline Parameter & \multicolumn{2}{l}{ NONMEM estimates $^{\mathrm{a}}$} & \\
\cline { 2 - 4 } & Point estimate & \%RSE & 95\% CI \\
\hline Intercept & -0.0190 & 494 & -0.203 to 0.165 \\
Effect of SS on SRI & 0.922 & 16.8 & $0.618-1.23$ \\
Effect of proteinuria on SRI & -0.468 & 49.1 & -0.919 to -0.0172 \\
Effect of RACE3 on SRI & -0.840 & 34.9 & -1.41 to -0.266 \\
Effect of RACE4 on SRI & 1.10 & 41.7 & $0.200-2.00$ \\
\hline
\end{tabular}

$C I$ confidence interval, $R A C E 3$ African American, $R A C E 4$ American Indian or Alaska Native, $R S E$ relative standard error, SRI systemic lupus erythematosus responder index, SS SELENA-SLEDAI

${ }^{a}$ All the covariate effects were evaluated on subjects receiving belimumab

${ }^{\mathrm{b}}$ Calculated as (standard error/parameter estimate) $\times 100 \%$ value $=-1.25$ ) or with a power model (change in objective function value $=-2.10$ ).

The effects retained in the final model of the main analysis were also significant, with qualitatively similar parameter values in the final model of the sensitivity analysis, which was restricted to belimumab-treated patients. Furthermore, in the sensitivity analysis, the exposure effect was not statistically significant. The sensitivity analysis retained one additional predictor, namely baseline corticosteroid use increasing the likelihood of SRI response (removal of which resulted in a change in objective function value $=-4.53$, i.e., the effect was significant at the $\alpha=0.05$, but not at the $\alpha=0.01$ level).

\section{Discussion}

Population-pharmacokinetic model parameters for SC belimumab in this analysis were consistent with outcomes from the IV belimumab popPK analysis [7] and with 
Fig. 5 Systemic lupus erythematosus responder index odds ratios in the final logistic regression model. All covariate effects were added as a linear function. SELENA-SLEDAI Safety of Estrogens in Lupus Erythematosus National Assessment-Systemic Lupus Erythematosus

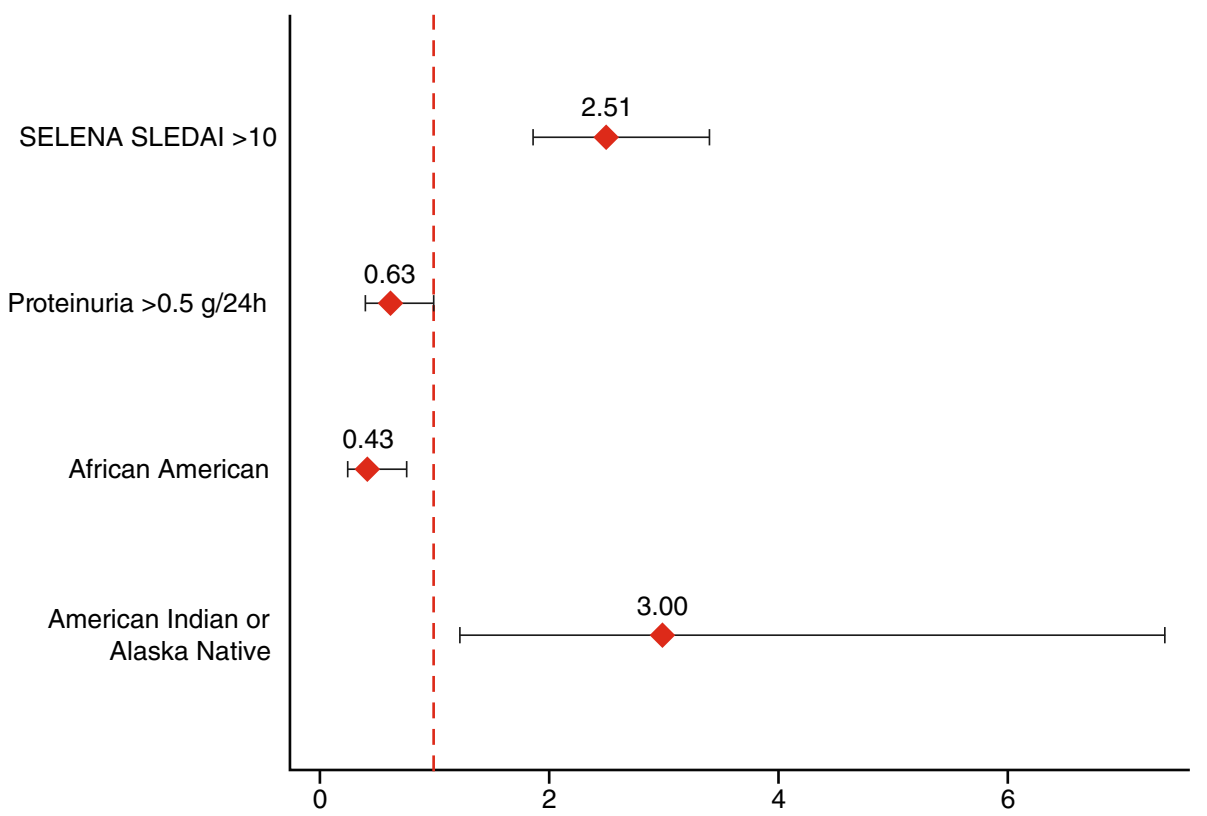

results reported for other IgG1 monoclonal antibodies without substantial target-mediated disposition [16]. The bioavailability of SC belimumab was estimated to be $74 \%$.

Subcutaneous belimumab $200 \mathrm{mg}$ QW in patients with SLE resulted in steady-state belimumab $C_{\text {avg }}$; similar to the $C_{\text {avg }}$ seen for IV $10-\mathrm{mg} / \mathrm{kg}$ dosing [7]. The efficacy of SC belimumab [10] is at least equivalent to that observed in the IV belimumab pivotal trials [4,5], with a similar safety profile. These results indicate that SC belimumab 200-mg QW dosing is suitable for SC administration in patients with SLE.

The derivation and analysis of the popPK model largely reflect phase III study data, which constitute the majority of the data analyzed. These data provide the primary evidence for the safety and efficacy of belimumab in patients with SLE, and consequently the model can be considered to reasonably predict the serum drug concentrations of SC belimumab expected in clinical practice.

No effects of patient age, sex, race, disease activity, comedications, or baseline characteristics were found to alter $\mathrm{SC}$ belimumab exposure in a manner to suggest that dose adjustments are required for maintenance of efficacy and safety in adults with SLE. Higher levels of baseline albumin were shown to decrease belimumab CL. A high albumin level may be an indicator of a high degree of neonatal $\mathrm{Fc}$ receptor $(\mathrm{FcRn})$ expression, leading to a high degree of protection of IgG and albumin from lysosomal degradation [17] and therefore also to reduced belimumab CL. By comparison, higher baseline IgG levels increased the CL of belimumab, likely owing to increased competition between endogenous IgG and belimumab for binding to saturable levels of FcRn. These two effects are not contradictory as albumin and IgG bind to different sites on FcRn and do not compete for binding with FcRn [17]. Therefore, albumin might be an indicator of the total capacity of FcRn-mediated recycling, while endogenous IgG levels indicate how much of that capacity can be used by exogenous $\mathrm{IgG}$, such as belimumab. These effects of albumin and $\mathrm{IgG}$ on belimumab $\mathrm{CL}$ are consistent with similar size effects identified in the earlier IV belimumab popPK analysis [7], and have also been previously observed for other monoclonal antibodies [16].

As expected, with BLyS (the target of belimumab) levels (median $1.31 \mathrm{ng} / \mathrm{mL}$ ) being several orders of magnitude lower than steady-state belimumab concentrations, no evidence for substantial target-mediated disposition of belimumab was found. The serially sampled PK profiles of the IV and SC phase I studies [7, 12, 14] reflect linear elimination kinetics and no substantial contribution of target-mediated clearance at the observed exposure concentrations. This was confirmed during the covariate analysis by the fact that neither BLyS levels nor other disease characteristics had a significant impact on belimumab clearance. However, target-mediated clearance at very low belimumab exposures only relevant in the late wash-out phase cannot be excluded.

The logistic regression analysis demonstrated that among the candidate effects, only baseline disease characteristics (high SLE disease activity, proteinuria) and two of the race categories were significant predictors of SRI response. These predictors are consistent with the results the subgroup analysis carried out as part of the statistical analysis for Study BEL112341 [10]; however, their clinical significance is not known. 
The analysis further demonstrated that SC belimumab exposure did not have a statistically significant impact on the SRI response or on the incidence of serious adverse events. This was consistent with a graphical exposure-response analysis for key biomarkers (naïve B cells, IgG, anti-double-stranded DNA antibodies, and complement) in Study BEL112341, which did not reveal a dependence of the biomarkers response at week 52 on $C_{\text {avg }}$ (data not shown). Moreover, these exposure response results for SC belimumab results are consistent with the dose response seen in the phase III IV belimumab studies [5, 7]. The $10-\mathrm{mg} / \mathrm{kg}$ dose groups were consistently superior across multiple efficacy endpoints compared with the $1-\mathrm{mg} / \mathrm{kg}$ dosing groups in the IV phase III studies, which led to the choice of the 200-mg weekly SC belimumab dose as the SC dose approximating the $C_{\text {avg }}$ for IV $10-\mathrm{mg} / \mathrm{kg}$ every 4 weeks dosing. However, the increase in efficacy for the $10-\mathrm{mg} / \mathrm{kg}$ IV dose was relatively moderate given the tenfold difference in dose, which is consistent with the interpretation that the 200-mg dose results in a range of exposures located in the shallow upper domain of a saturating exposure-efficacy response curve. Surprisingly, in the combined belimumab/placebo population of the BLISS-SC study, the odds ratio of SRI response was numerically improved for patients with SLE in the heavier body weight quartiles [10]. The SC belimumab popPK/PD analysis demonstrated that no dose adjustment is required based on body weight or other analyzed patient characteristics.

\section{Conclusion}

This analysis indicates that belimumab $200 \mathrm{mg}$ QW is appropriate for SC administration in patients with SLE and that no dose adjustments are required for adult patients to maintain efficacy and safety.

Acknowledgements Medical writing assistance was provided by Louisa Pettinger, PhD, and Jennie McLean, PhD, of Fishawack Indicia Ltd, and was funded by GlaxoSmithKline. The authors wish to thank the patients and trial investigators involved in this study.

\section{Compliance with Ethical Standards}

Funding This study was funded by GlaxoSmithKline.

Conflict of Interest Herbert Struemper is a former employee of and owns stock in GlaxoSmithKline. David Roth is an employee of and owns stock in GlaxoSmithKline. Mita Thapar has no conflicts of interest directly relevant to the contents of this study.

Ethics Approval All procedures performed in studies involving human participants were in accordance with the ethical standards of the investigational review board or human subjects committee and with the 1964 Helsinki Declaration and its later amendments or comparable ethical standards.

Consent to Participate Informed consent was obtained from all individual participants included in the study.

Open Access This article is distributed under the terms of the Creative Commons Attribution-NonCommercial 4.0 International License (http://creativecommons.org/licenses/by-nc/4.0/), which permits any noncommercial use, distribution, and reproduction in any medium, provided you give appropriate credit to the original author(s) and the source, provide a link to the Creative Commons license, and indicate if changes were made.

\section{References}

1. Adinolfi A, Valentini E, Calabresi E, Tesei G, Signorini V, Barsotti S, et al. One year in review 2016: systemic lupus erythematosus. Clin Exp Rheumatol. 2016;34(4):569-74.

2. Cancro MP, D'Cruz DP, Khamashta MA. The role of B lymphocyte stimulator (BLyS) in systemic lupus erythematosus. J Clin Invest. 2009;119(5):1066-73.

3. Baker KP, Edwards BM, Main SH, Choi GH, Wager RE, Halpern WG, et al. Generation and characterization of LymphoStat-B, a human monoclonal antibody that antagonizes the bioactivities of B lymphocyte stimulator. Arthritis Rheumatol. 2003;48(11):3253-65.

4. Furie R, Petri M, Zamani O, Cervera R, Wallace DJ, Tegzova D, et al. A phase III, randomized, placebo-controlled study of belimumab, a monoclonal antibody that inhibits B lymphocyte stimulator, in patients with systemic lupus erythematosus. Arthritis Rheum. 2011;63(12):3918-30.

5. Navarra SV, Guzman RM, Gallacher AE, Hall S, Levy RA, Jimenez RE, et al. Efficacy and safety of belimumab in patients with active systemic lupus erythematosus: a randomised, placebo-controlled, phase 3 trial. Lancet. 2011;377(9767):721-31.

6. GlaxoSmithKline. Benlysta prescribing information. http://www. gsksource.com/gskprm/htdocs/documents/BENLYSTA-PI-MG. PDF. Accessed 3 Aug 2017.

7. Struemper H, Chen C, Cai W. Population pharmacokinetics of belimumab following intravenous administration in patients with systemic lupus erythematosus. J Clin Pharmacol. 2013;53(7):711-20.

8. Furie R, Stohl W, Ginzler EM, Becker M, Mishra N, Chatham W, et al. Biologic activity and safety of belimumab, a neutralizing anti-B-lymphocyte stimulator (BLyS) monoclonal antibody: a phase I trial in patients with systemic lupus erythematosus. Arthritis Res Ther. 2008;10(5):R109.

9. Wallace DJ, Stohl W, Furie RA, Lisse JR, McKay JD, Merrill JT, et al. A phase II, randomized, double-blind, placebo-controlled, dose-ranging study of belimumab in patients with active systemic lupus erythematosus. Arthritis Rheumatol. 2009;61(9):1168-78.

10. Stohl W, Schwarting A, Okada M, Scheinberg M, Doria A, Hammer AE, et al. Efficacy and safety of subcutaneous belimumab in systemic lupus erythematosus: a fifty-two-week randomized, double-blind, placebo-controlled study. Arthritis Rheumatol. 2017;69(5):1016-27.

11. Sheikh SZ, Hammer AE, Fox NL, Groark J, Struemper H, Roth D, et al. Evaluation of a novel autoinjector for subcutaneous selfadministration of belimumab in systemic lupus erythematosus. Int J Clin Pharmacol Ther. 2016;54(11):914.

12. Shida Y, Takahashi N, Sakamoto T, Ino H, Endo A, Hirama T. The pharmacokinetics and safety profiles of belimumab after 
single subcutaneous and intravenous doses in healthy Japanese volunteers. J Clin Pharm Ther. 2014;39(1):97-101.

13. Yapa SW, Roth D, Gordon D, Struemper H. Comparison of intravenous and subcutaneous exposure supporting dose selection of subcutaneous belimumab systemic lupus erythematosus phase 3 program. Lupus. 2016;25(13):1448-55.

14. Cai WW, Fiscella M, Chen C, Zhong ZJ, Freimuth WW, Subich DC. Bioavailability, pharmacokinetics, and safety of belimumab administered subcutaneously in healthy subjects. Clin Pharmacol Drug Dev. 2013;2(4):349-57.
15. Chilton F, Collett RA. Treatment choices, preferences and decision-making by patients with rheumatoid arthritis. Musculoskelet Care. 2008;6(1):1-14.

16. Dirks NL, Meibohm B. Population pharmacokinetics of therapeutic monoclonal antibodies. Clin Pharmacokinet 2010;49(10):633-59.

17. Chaudhury C, Mehnaz S, Robinson JM, Hayton WL, Pearl DK, Roopenian DC, et al. The major histocompatibility complex-related $\mathrm{Fc}$ receptor for $\mathrm{IgG}(\mathrm{FcRn})$ binds albumin and prolongs its lifespan. J Exp Med. 2003;197(3):315-22. 